\title{
Automated operant assessments of Huntington's disease mouse models
}

Emma Yhnell ${ }^{1}$ and Andreas Heuer ${ }^{2}$

${ }^{1}$ Neuroscience and Mental Health Research Institute, Cardiff University, Cardiff, Wales, UK

${ }^{2}$ Molecular Neuromodulation, Experimental Medicine, Lund University, Lund, Sweden

Email address: YhnellE@cardiff.ac.uk

Telephone: + 4402920688456

Running Head: Operant assessments of HD mice 


\section{i. Summary/Abstract}

Huntington's disease (HD) presents clinically with a triad of motor, cognitive, and psychiatric symptoms. Cognitive symptoms often occur early within the disease progression, prior to the onset of motor symptoms, and they are significantly burdensome to people who are affected by HD. In order to determine the suitability of mouse models of HD in recapitulating the human condition, these models must be behaviourally tested and characterised. Operant behavioural testing offers an automated and objective method of behaviourally profiling motor, cognitive and psychiatric dysfunction in HD mice. Furthermore, operant testing can also be employed to determine any behavioural changes observed after any associated interventions or experimental therapeutics. We here present an overview on the most commonly used operant behavioural tests to dissociate motor, cognitive, and psychiatric aspects of mouse models of Huntington's disease.

\section{ii. Key Words}

Huntington's disease, mouse model, knock-in, transgenic, cognition, behaviour, operant, 9hole box, Skinner box, touch screen 


\section{Introduction}

Since the identification of the mutant huntingtin gene which causes Huntington's disease (HD) [1], a vast number of genetically modified animal models have been created. These animal models range from large animal models such as sheep [2], pigs [3] and monkeys (Macaca mulatta) [4], to smaller animal models such as rats [5], Caenorhabditis elegans [6] and cellular models [7]. The range of animal models of HD available is presented and discussed in other Chapters of this textbook. However, by far the most widely generated and characterised animal model of HD is the genetically modified mouse [8].

The focus of this chapter is the operant analysis of phenotypic behaviour in HD mouse models, which make up a small part of the tests available. For the interested reader we would like to refer to further research on non-operant behavioural testing [9-14] and excellent reviews of behavioural phenotyping in $\mathrm{HD}$ mice $[15,16]$, which provide an overview of non-operant assessments of motor as well as non-motor functions in mouse models of HD and cover many topics including; depression, anxiety, fear conditioning, sensory-motor gaiting, as well as learning, spatial navigation, metabolic disturbances and sleep patterns. An extensive and detailed description of the large number of mouse models of HD which are available for use in scientific research is given in other Chapters in this volume. However, it should be noted that the ease of genetic manipulation within the mouse, the heavily related genome to humans [17], the short gestation period and relatively low cost of housing, makes the mouse an ideal organism for modelling genetic diseases such as HD.

As HD is a human disease, it is anticipated that the available animal models will only recapitulate certain aspects of the disease and therefore, care should be taken that the 
appropriate model is chosen. For mouse models to recapitulate the human condition of HD, they must be behaviourally tested to determine their predictive, face, and construct validity. Due to the large number of mouse models of HD that are available for use in research, the importance of sensitive behavioural tests to determine if a mouse model replicates some or all of the behavioural symptoms observed in the human condition is essential.

In line with this, the human condition of HD is a progressive neurodegenerative disorder and therefore it is of importance to consider the appropriate time-point of operant behavioural testing. A stable and consistent behavioural readout in combination with appropriate matched control groups is essential for meaningful comparisons to be made. Within each model the loci of disease pathology and the resulting behavioural, cognitive and psychiatric impairments are likely to match the affected underlying brain circuitry. For example, early in the disease affected functions are short term memory, attention, cognitive flexibility, reversal learning and set-shifting, as well as impulse control and perseverative responding which all correspond to a pathology in related brain areas associated with the fronto-striatal circuitry.

As a progressive disorder, repeated testing over the life span of the respective animal model is useful to distinguish behavioural phases which are comparable to the asymptomatic, presymptomatic, or symptomatic stages of HD in people. In order to create operant tests which appropriately assess cognitive behaviours in mouse models of HD, the behavioural symptoms observed in the human condition of HD need to be fully understood. HD is perhaps traditionally seen as a motor condition, due to the presentation of chorea and involuntary jerking or writhing movements often in the later stages of the disease [20, 21]. However, as our understanding of HD has developed, it is now becoming increasingly clear that cognitive 
symptoms often occur prior to the onset of motor symptoms [22-29]. The precise cognitive symptoms of HD may vary between individuals, although they can often include alterations in: perseverance [30, 31], set shifting [23], reversal learning [32], alternation [24], judgement and risk taking [30], cognitive flexibility [33], as well as facial and emotional recognition [3436].

Furthermore, alterations in cognitive function have been shown to significantly affect daily activities, independence and quality of life, even during the early stages of the disease [37, 38]. Importantly, cognitive function is often used as an indicator of an individual's ability to manage independently [39] and therefore alterations in cognitive function can significantly impact on the quality of life of people living with HD. Furthermore, alterations in psychiatric function have been frequently reported $[\mathbf{3 0}, \mathbf{4 0}, \mathbf{4 1}]$, including symptoms such as aggression [42], irritability [43], apathy [44] and most commonly depression [45, 46].

For the application of HD mouse models, many hand testing procedures have been developed to assess one or several aspects of the behavioural measures stated above, utilising a variety of different behavioural equipment including mazes. Although these tests have revealed many insights into the nature and progression of the cognitive and psychiatric deficits that emerge over the time course of the disease, they are discussed elsewhere [9-13, 47]. The disadvantages of hand testing procedures, such as these, can be the introduction of observer bias, they are highly time-consuming, and they do not allow the simultaneous assessment of multiple parameters.

Operant assessment of behaviour has the distinct advantage that data collection is automated (eliminating observer bias) as well as the simultaneous collection of multiple parameters (e.g. reaction times, movement times, response bias, accuracy, etc.), which 
allows for the dissociation of cognitive, psychiatric, and motor aspects of performance. Furthermore, when compared to behavioural hand testing, such as mazes, operant testing is comparatively rapid as it allows multiple animals to be tested simultaneously, generating large volumes of data with minimal experimenter input. Depending on the nature of the operant task, typically $80-120$ trials can be collected within a 30 -minute testing session. The parametric design of the tests and multivariate nature of the data allow for the detection of small effects that may be missed in more crude hand testing approaches. Furthermore, tasks can specifically be designed to probe certain functions directly, thereby providing a rationale for therapeutic interventions.

When designing, and conducting operant tasks in HD mice it is important to consider which behavioural symptoms they are specifically designed to test. For example, while a task may be designed to predominantly test executive function by recording accuracy of responses in the 5-choice serial reaction time task, additional behavioural measures of response time and reaction time can be collected as readouts of motor function. Furthermore, the number of errors or perseverative responses made can be recorded as further measures of HD related behaviours. However, it is important to note that many factors can mask or influence the results of behavioural testing and when characterising new mouse models of $H D$, several tests should be conducted in parallel to ensure an impairment is not due to external factors (i.e. macular degeneration would mask deficits on the choice reaction time task due to visual impairment, which could be easily revealed by performing a light-dark transfer test [48]. There are many alternative operant test designs which can be utilised for testing HD mice. Operant tests are designed to test specific and discrete motor, cognitive and psychiatric functions, a selection of which will be described below. 


\section{Materials}

Operant analysis of mouse behaviour allows the generation of tasks that probe specific cognitive functions which then can serve as a basis for therapeutic readouts. The operant experimental apparatus utilised can vary considerably between studies. In fact, a direct comparison of the 9-hole operant box and the Skinner operant box demonstrated that the behavioural results observed critically depend on the type of operant apparatus used [49]. Therefore, it is important to consider which specific operant apparatus or configuration of apparatus is to be utilised in any given study. The equipment can be purchased from various suppliers that offer complete testing packages. The operant programs can usually be purchased or easily adapted from online repositories and therefore relatively little programming skills are required. In addition, fellow scientists and colleagues often provide invaluable knowledge and help regarding specific operant programmes. If there is interest in developing new tasks or in combining operant testing with other technologies, i.e. optogenetics, electrochemistry, calcium imaging, etc., learning how to create specific operant programmes can be advantageous.

The operant testing equipment requires basic maintenance and cleaning, although it is robust when treated with the necessary care. Basic soldering skills will aid in the maintenance and replacement of smaller parts. Furthermore, the supplier will support and facilitate the replacement of larger parts if this is necessary. The operation of the operant behavioural testing system is simple, requires minimal training, and little prior experience. 


\subsection{The Skinner operant box}

The Skinner operant box typically contains two retractable response levers located either side of a hinged Perspex panel, which sits in front of a magazine to enable the delivery of reward [50]. A stimulus light is located above each lever. The apparatus may also contain a house light and typically chambers are enclosed in sound-attenuating boxes. Alternative operandi can consist of keychains to pull, wheels to turn, etc.

Modified versions of the traditional Skinner box also exist, these often contain response holes rather than levers, located either side of the reward magazine (as illustrated in Figure 1D). These modified versions with response holes may be easier for rodents to respond into, as a nose poke response may be more naturalistic for rodents than a lever press [49]. Furthermore, motor problems can be apparent in HD mice, which may make lever responding comparatively more difficult than nose poking [51].

(Figure 1 near here)

\subsection{The 9-hole operant box}

The 9-hole operant box was originally utilised to test visual attention in the rat $[\mathbf{5 2}, \mathbf{5 3}]$. Subsequently it has been further utilised to probe attentional function in the mouse $[\mathbf{5 4}, \mathbf{5 5}]$ and it has specifically been applied to measure attention in HD mouse models $[56,57]$.

The 9-hole operant boxes (Figure 1A) are controlled by an external computer operating system. Each operant box typically constitutes a sound attenuating chamber which encloses the 9-hole box made of aluminium on all sides with a clear Perspex lid. The rear wall of each chamber is curved and contains a horizontal array of nine holes. Each hole contains 
photocell infrared beams, localised at the front of each hole to detect nose pokes. At the rear of each hole a white light-emitting device (LED) acts as the target visual stimulus.

Reward is ordinarily delivered utilising a peristaltic pump, which delivers a liquid reward to a magazine at the front of the operant box immediately opposite the 9-hole array (Figure 1B). The reward delivery to the magazine is signalled by an LED located above the magazine and nose entry into the magazine is detected by infrared beams located across the opening of the magazine. 'House lights' are also located on the side walls of the operant chamber. The experimental set up of the 9-hole box offers a degree of flexibility in the operant tasks which can be designed and performed due to the 9 holes which are available within the operant programme (Figure 1C).

\subsection{The operant touch screen apparatus}

Recently, operant boxes fitted with touch screens have been introduced and successfully adapted for use in rodents [58]. As a comparatively recent development the published literature on the use of this specialised equipment is still sparse. However, the obvious advantage of using a screen for stimulus presentation, rather than a focal light source, lies in the ability to deliver virtually any type of visual stimulation to the animal, hence providing a higher degree of flexibility. This can potentially lead to the development of tasks that assess cognitive set shifting. Furthermore, there are clear advantages for the translational use of touch screens into primate and clinical studies.

The touch screen operant apparatus set-up follows the general design of both the Skinner and 9-hole operant boxes with the screen located at the far end of the chamber and the reward delivery panel located at the opposite end. In the first adaptation for mice, this configuration has been used to demonstrate a two-choice reversal learning procedure in the 
R6/2 mouse model of HD [59]. Despite the automated nature of the apparatus, the learning of a simple reversal discrimination task, takes considerably longer compared to the learning of the traditional nose-poke response. The strength of the touch screen apparatus lies in the ability to utilise the screen in a way that stimuli can be presented in two dimensions (i.e. location discrimination task).

Several tasks have been adapted and developed, mostly by the groups of Tim Bussey and Lisa Saksida for use in rodents, such as the location discrimination learning task in mice [60], the pattern separation task in rats [61] and the delayed nonmatching to location task in mice [62]. Operant touch screen apparatus provides an exciting new piece of highly specialised equipment that is important to consider among other automated testing procedures, especially when testing cognitive functions that require the presentations of more complex visual stimuli.

In summary, each operant apparatus has distinct advantages, the 9-hole box for example offers the possibility to present the stimulus-response location over a wide spatial array. Furthermore, the nose-poke response is a more natural behaviour to the mouse than a lever press and may therefore facilitate faster learning. In the retractable lever, Skinner Box apparatus, the levers can be presented only during certain task intervals, which facilitates discrete trial designs and prevents inappropriate responding during inter-trial or time out intervals. Although the touch screen apparatus is the most recent development for methods in operant behavioural testing, for most tasks the classic Skinner box or 9-hole box operant configurations will suffice. 


\section{Methods}

There is a growing volume of literature on operant tests for HD mice, which is summarised in Table 1. The individual steps necessary for operant testing in rodents are detailed below. The background strain of mouse (see Note 1) to be used as well as the use of appropriate control groups (See Note 2) should be carefully considered in the experimental set up.

\subsection{Protocol for training}

\subsubsection{Restriction regimes}

Food or water restriction regimes are typically utilised in operant testing to promote learning and to ensure that all animals are restricted to the same degree. The choice of either food or restriction and the comparative benefits and drawbacks of each will be further discussed below in the Notes section, see Note 3.

The precise details of the restriction regime employed should comply with the relevant legal local restrictions, guidelines, and practices. However, it is recommended that the restriction regime should begin at least 5 days before the start of operant training to achieve a stable baseline of weight. Depending on the licence we recommend free access to water for three hours per day after operant training or the provision of weighed amounts of food 1-2 hours after the end of respective training session, respectively. Body weight should be monitored daily and the restriction adjusted accordingly. Common practice is to keep the animals at $85-90 \%$ of their free feeding weight (whilst allowing for natural growth; growth curves can be maintained).

The choice of reinforcing reward can vary among different studies. Several reinforcers have been described in the literature, e.g. solid reinforcers such as sucrose or grain 
pellets and liquid reinforcers such as sucrose, condensed milk, or strawberry milk, although the reinforcer will typically contain some calorific value. The reinforcer should be gradually introduced into the home cage for several days prior to the beginning of operant testing to prevent neophobic response. We and others routinely use $5 \mathrm{uL}-10 \mathrm{uL}$ of strawberry milk (Yazoo ${ }^{\circledR}$ ) per rewarded trial, which allows for a sufficient number of responses in a 30 to 60 minute session without satiating the animals [55]. Special care should be taken to appropriately clean the apparatus (peristaltic pumps and tubing) to facilitate liquid reward delivery, as milk-based rewards can easily block the tubing. To prevent blocking of the tubes or the growth of microbes, we recommend flushing the tubes daily after use with lukewarm water, subsequently with $70 \%$ ethanol, and finally with water.

\subsubsection{Training}

Mice require training in order to respond appropriately in the operant box. Typically, mice require training until they respond to a particular level or pre-set criterion. We recommend moving the animals on to the next stage of training when they perform with asymptotic performance. The mice should be habituated to the testing environment for at least one hour before beginning operant testing.

The training protocol for most tasks then consists of stepwise training to the full task. Training is usually broken down over several days:

\subsubsection{Daily preparations:}

1. Prepare the equipment and boxes by turning on the computer equipment.

2. Open fresh strawberry milk and load the peristaltic pumps. Ensure that the tubing is not clogged and that no air bubbles are in the tubing. Clear polyethylene tubing 
allows for the easy visualization of any blockages. Ensure that the reward is delivered into the magazine: this can easily be done by running a test program on the computer to ensure all of the equipment works as intended. We use a program that illuminates all response holes and delivers a reward if a nose poke is detected. The aims of this are three-fold to ensure: (i) the stimulus lights are working appropriately; (ii) the photocell detectors are able to appropriately detect a beam break (= nose poke); and (iii) reward delivery occurs as expected.

3. The setting up and testing of the equipment can be completed whilst the animals habituate to the testing environment.

4. Load the respective computer program.

5. Place animals into the operant boxes.

6. Start the selected operant program on the computer.

7. Data will save automatically at the end of the testing programme.

8. Ensure all equipment is appropriately cleaned at the end of the testing session, including flushing through the peristatic pumps.

\subsubsection{Day 1: Habituation}

The mouse is placed in the operant chamber with house light on and $100 \mathrm{uL}$ of reward is placed into the reward magazine. The mouse is allowed 20 minutes to explore and consume the reward ad libitum.

\subsubsection{Day 2: Magazine training}

The mouse is placed in the operant chamber and a non-contingent delivery of reward is utilised whereby the animal must enter the illuminated reward magazine to retrieve the 
reward. The session typically lasts for either for 20 minutes or until 100 rewards have been consumed.

\subsubsection{Days 3-5: Nose poke hole training}

At the start of each trial the central response hole is illuminated whilst all other lights remain extinguished. A nose-poke response into the illuminated hole will result in the extinguishing of the lit hole, the illumination of the magazine light and the simultaneous delivery of a reward into the magazine. After the reward is collected, the magazine light is switched off and the house-light is switched on for 3 seconds as an inter-trial interval before the next trial commences as described above. To promote the learning of this procedure the response hole can be painted with the reinforcer, such as strawberry milk, to encourage responding. For this, a fine paint-brush can be used to place a small amount of strawberry milk just behind the photocell detector. Care should be taken not to block the photocell detector, as this will interfere with data acquisition.

\subsubsection{Day 6 onwards:}

After mice acquire a steady rate of responding any further training is dependent on the specific operant task. In general, it is recommended that animals reach an appropriate level of performance before moving the animals to the next step of the task. On the 5choice serial reaction time task for example the stimulus length can be reduced over successive sessions from 10 seconds to 0.5 seconds. On each training day the performance should be monitored to ensure that performance is just approaching asymptote. During the training and subsequent testing phases, a large volume of data will be generated via the operant system, see Note 4 . 


\subsection{Alternative test designs and schedules}

\subsubsection{Fixed ratio schedule}

In the fixed ratio operant test $[55,57]$, mice are required to poke into the central hole of the 9-hole array in response to illumination or manipulate the presented operandum as required (i.e. lever press, chain pull, etc.), to obtain reward. The number of responses is recorded. The size of the reward can be varied to investigate the acquisition and amenability in responding. The reward ratio can be changed, i.e. 1 press equals 1 reward delivery (FR1) or 5 presses result in reward delivery (FR5). When a one press equals one reward ratio is used the terminology FR1-schedule or continuous reinforcement (CRF) is usually used interchangeably. By comparing the performance on the various ratio tasks one can determine the motivation (or willingness to work) for a given reward.

\subsubsection{Progressive ratio schedule}

Progressive ratio operant tasks are designed to test the motivation of mice, in terms of how many responses they are prepared to make to obtain a given level of reward before ceasing to respond. The schedule of reinforcement requires progressively more responses, to be made on each successive trial. However, the precise schedule of responding and requirements and ratio of responding can differ considerably between studies. When animals cease to respond for a pre-defined period of time, termed the break point, this is recorded.

Whereas many operant tasks allow the animals to perform on discrete trials (each trial has a binary endpoint where the animal is either rewarded or punished), ratio tasks, such as the progressive ratio task, are considered as 'free operant', i.e. the animal can respond at any 
time and is rewarded according to a defined schedule of reinforcement. These range from continuous reinforcement to more complex reinforcement schedules. In the fixed reinforcement schedules a particular number of correct responses leads to a reward whereas progressive reinforcement schedules require an alternation to the response during the test session.

The progressive ratio task assesses a form of cost-benefit ratio, i.e. how hard the animal is prepared to work for a given reward. In its simplest form, a single response results in a reward on the first trial, whereas two responses are required on the second trial, three responses on the third trial, and so on. Subjects usually respond until they reach a "breakpoint", i.e. the level of satiation, where they are no longer motivated to work for a given reward. It is a very simple but also a very powerful paradigm as testing can be conducted over relatively few days. Many parameters can be changed, for example increasing the ratio (i.e. $1,1,1,3,3,3,6,6,6, \ldots n, n, n$ or $1,1,1,5,5,5,10,10,10, \ldots n, n, n$ ) or changing the reward size (i.e. more or less). The progressive ratio task has been successfully employed utilising both the $\mathrm{Hdh}^{\mathrm{Q} 92}$ and $\mathrm{Hdh}^{\mathrm{Q} 111} \mathrm{HD}$ mouse $[57,63]$. The definition of the "break-point" has proven somewhat arbitrary as mice will never stop responding fully and the occasional response is usually made even with long intervals. Researchers have used several "break-points" and it is recommended to include these into the data collection software, i.e. record the ratio an animal breaks for 1 minute, 3 minutes, 5 minutes, etc.

\subsubsection{Five choice serial reaction time task (5-CSRTT)}

The 5-choice serial reaction time task (5-CSRTT) was originally developed to test attentional function in rats [52], since then it has been modified for use in mice [54]. The 5-CSRTT has 
been used in the behavioural profiling of both the $\mathrm{Hdh}^{\mathrm{Q} 92}$ and $\mathrm{Hdh}^{\mathrm{Q} 111}$ mouse models [56, 57].

In the 5-CSRTT animals are trained to respond to a stimulus which is presented randomly across a 5-hole light array, therefore for this specific task the 9-hole operant box apparatus is required (Figure 1C). The purpose of this task is to investigate attentional performance and spatial awareness, akin to vigilance tests in humans. The stimulus length can be reduced to increase the difficultly and attentional load of the task. A limited hold time can be applied to the task, whereby if the animal does not respond during the stimulus length or in a subsequent length of time the stimulus light is extinguished and a 'time out' period is applied by illumination of the house light.

\subsubsection{Serial implicit learning task}

The serial implicit learning task (SILT), and more recently the extended sequence learning task (ESLet) [64] are based on the 5-CSRTT paradigm, although they have been modified to probe implicit sequence learning. In the SILT, animals are trained to respond to a 2-step sequence of stimulus lights in order to receive a reward. A continuous stimulus light is randomly presented across the array (as in the 5-CSRTT). A correct response to the first stimulus light (S1) results in the simultaneous extinguishing of the light stimulus and illumination of a second light (S2). A correct response to S2 results in delivery of a reward into the magazine. However, a predictable stimulus sequence is embedded among other unpredictable sequences to probe implicit learning. Various parameters can be assessed, such as step size (i.e. the distance between the two consecutive responses, movement and reaction times as well as response accuracies towards the respective stimulus and stimulus sequence. Despite an equal distance between the randomised response sequence and the 
predictable stimulus sequence, the predictable sequence should be performed faster and with greater accuracy (as an indication of implicit learning). This task requires relatively intensive training, although it is one of the first approaches to measure implicit learning in operant boxes for mice.

\subsubsection{Delayed alternation (DA) task}

The delayed alternation task is a classical operant test which was originally designed to study fronto-striatal circuitry in monkeys $[65,66]$. It has since been translated for use in automated boxes for rats by Dunnett [67] and recently been adapted for use in mouse models [68]. In brief, the subject is presented with a choice of two response options (response holes, lever presses, etc.). At the beginning of the first trial a response to either option will result in the delivery of a reward. After a variable inter trial interval both response options are presented again. Now only a response on the opposite response option will result in reward delivery, whilst choosing the same option will result in a time out. The response rule for successful performance is thus to alternate responding continuously between the two response options. To assess working memory the variable interval is, randomly chosen by the computer programme, of different durations between the two successive responses. As a result of introducing increasing delays, the accuracy of performance usually decreases with increasing delays, i.e. following a decay function. Performance where short delays are utilised is usually highly accurate, whereas performance where long delay periods are introduced approaches chance levels [68]. See Note 5 for further explanation of the mediating strategies which have been utilised by rodents in performing this task. 


\subsubsection{Delayed matching and delayed non-matching to position (DMTP/DNMTP) task}

The delayed matching to position and delayed non-matching to position (DMTP and DNMTP) operant tasks, are designed to test working memory and reversal learning. The DMTP/DNMTP tasks were originally developed in rats [69] in the Skinner box apparatus, although they have since been modified and used in HD mice [51]. In the DMTP task the animal is required to respond to a stimulus; the animal is then re-centralised before being presented with two simultaneous stimuli. In the DMTP task the animal is required to match the second response with the stimulus that it previously responded to obtain a reward. In the reversal of the task, DNMTP, the animal is required to respond into the alternative stimulus to which it had not previously responded to obtain reward.

\subsubsection{Delayed matching and non-matching to sample (DMTS/DNMTS) task}

The delayed (non) matching to sample task is conceptually similar to the (non) matching to position as it also requires a memory component to identify the location of the second stimulus. In the (non) matching to sample procedure a stimulus (the sample) is presented to the animal and after a variable delay the same stimulus is presented in different locations [70]. The matching of the stimulus is irrespective of the location or response and just depends on the nature of the stimulus. This task has the distinct advantage that mediating response strategies (e.g. sitting at the correct location, therefore not depending on a memory component) can be avoided. 3.2.8 Comparison of delayed matching tasks

The delayed matching to sample task is a delayed choice task [71], which is similar to both, the delayed alternation task and delayed matching to position/sample tasks,. First a sample response option, e.g. a response lever. After a positive response, a delay period is initiated after which the subject enters the choice phase. Of the two response options the subject can 
chose from one which will result in reward delivery whereas the other will result in a time out period. In the delayed matching to sample variant a response has to match the sample option whereas in the delayed matching to position variant the response has to match the sample location. In contrast to the delayed alternation procedures in the DMTP/DNMTP and DMTS/DNMTS it is possible to reverse the response rule, whereas in the delayed nonmatching configuration the subject has to press the opposite response than the sample option. This reversal of the response rule is crucial as it can be used to assess learning, cognitive flexibility, perseveration and other cognitive measures which are of interest in HD.

\subsubsection{The 'Carli' task (lateralised reaction time task)}

Choice response tasks, such as the Carli task, have been very powerful in separating reaction and movement times and they allow for the assessment of multiple parameters simultaneously. The lateralised choice reaction time task (also known as the "Carli task") is a stimulus-response task first used to assess the effects of dopaminergic lesions in rats [72]. The task has been used in rats in the Skinner box apparatus as well as the 9-hole box operant chamber $[\mathbf{7 3}, \mathbf{7 4}]$. In brief, the test subject must sustain a nose poke in a centralised response location for a variable delay. After this delay period, has elapsed a brief stimulus is presented randomly to either side of the animal's head. After stimulus detection, the rodent then has to withdraw its nose from the centre location (=reaction time) and report the occurrence of the lateralised stimulus by poking/pressing the lateralised lever/hole.

Different configurations of this test have been used to demonstrate that striatal dopaminergic differentiation does not cause a primary sensory deficit (as equal performance

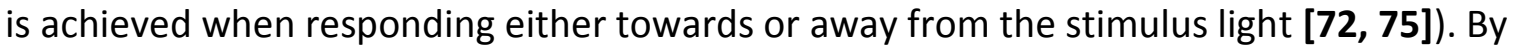
testing animals on one side on alternating days but with a near and a far response location 
the animals response bias could be assessed in further detail [49]. Furthermore, this test has been used in animals with excitotoxic lesions (QA) to the striatum as a unilateral model of HD to demonstrate response bias in rats [74] as well as the learning to use the transplant in cell replacement therapy models [76].

The operant tasks described above are often employed in a longitudinal manner to explore the development and progression of HD related cognitive symptoms over time. However, repeated testing in operant tasks of this nature has been shown to modify the associated disease phenotype [18]. This is important to consider in the interpretation of the observed results, see Note 6 .

\subsection{Conclusions}

Operant testing offers a highly sensitive, rapid and automated way of behaviourally testing cognition in HD mice. However, when used in combination with other behavioural tests it can provide a comparatively more extensive characterisation of behavioural profiles.

Operant testing has been used as a therapeutic intervention in HD mice which modifies disease related symptoms $[\mathbf{1 8}, \mathbf{1 9}]$. Therefore, the future use of operant training or testing in HD mice may well be as part of a combinatorial therapy when translated into the patient clinic. Furthermore, recent technological developments allow for the active probing of genetically defined cell populations (e.g. optogenetics or chemogenetics) as well as simultaneously for the selective recordings of cell activity (e.g. calcium imaging, electrochemistry). It will be interesting to observe how these technologies are combined with operant testing to further explore associated cognitive mechanisms in both healthy and diseased brain.

\section{Notes}


1. Choosing a mouse model of HD heavily depends on the research question being asked, as there are a variety of models available, each with their relative strengths and weaknesses. As there is no "best model" or "best behavioural test", the subjecttest combination should be carefully considered and chosen prior to the commencement of the study. One important factor to consider is the background strain of mouse to be used, as some strains response to training differently to others. The most commonly used strain for behavioural studies is on a C57/BI6 background. Other strains have been described to suffer from retinal degeneration or are generally more difficult to train (e.g. SV129) [77]. Furthermore, the background strain of the mouse model has been shown to significantly influence HD related behaviours [77]. To make operant analysis comparable it is advisable to use the same background strain, in our experience, the C57/B 6 strain has been shown to perform well on operant tasks.

2. Appropriate control groups should also be utilised during operant testing, which may consist of homozygote heterozygote and wild-type animals of both genders. Furthermore, the specific genetic construct utilised will significantly affect the behavioural phenotype and thus the observed results. Finally, the nature of the operant apparatus used [49], including the experimental set-up of the operant apparatus [51], will influence the results obtained.

3. In order to motivate mice to respond appropriately to obtain reward, food or water restriction regimes are often utilised. It is important to consider that the exact protocols of food or water restriction can vary significantly between studies. However, a direct comparison of food or water restriction protocols in C57BL/6J mice 
[78] suggested that, as measured by body weight fluctuations, mice tolerate water restriction comparably better than food restriction. Furthermore, behavioural measures of exploration, memory and social dominance differed based on the restriction applied to the mice. Perhaps most notably, in the conditional learning operant paradigm used, there were significant differences in the number of cumulative nose pokes demonstrated between mice that had been food restricted in comparison to those who had been water restricted. Furthermore, specifically in HD mice it has been shown that calorie restriction can modify disease progression and prolong life [79]. Therefore, the associated effects of applying food or water restrictions during operant testing, require careful consideration in HD mice.

4. Operant behavioural testing has the potential to produce large volumes of data, every animal can produce $60-120$ responses in a 30-minute session, depending on the task. With multiple days of testing the amount of data accumulates rapidly and therefore a good knowledge of a spreadsheet software to appropriately manage the data that is produced is advised. In addition, statistical analysis of the associated data can become complex with multivariate, repeated-measures designs. Therefore, good knowledge of a statistical software package that allows for multifactorial analysis is recommended for data analysis.

5. Although operant tasks are often designed to test specific cognitive domains, there are some outputs in the results that can be interpreted independent of the specific task. For example, response time measures and the ability to respond when the stimulus length is decreased or the ability to respond when the rule is changed. Rodents have been shown to use mediating strategies, which can lead to the 
misinterpretation of results. When assessing rats on a delayed alternation task it has been found that they were simply waiting in front of the "to-be-pressed" lever, rather than using their working memory to remember the correct response location. Only by observing the animals whilst conducting the task can these behaviours be identified. In the present example, a simple centralising nose-poke to initiate the next trial in the rear wall of the operant chamber allowed to abolish the mediating response [70].

6. The longitudinal assessment of mice utilising multiple behavioural time points requires large numbers of animals and the resources to be able to appropriately housed and test large numbers of animals. This experimental design can increase costs and be extremely labour intensive. Most common is the staggered approach, in which cohorts are tested in intervals so that the daily workload is manageable, although this will extend the total duration of the study. In addition, a cohort should extend throughout the longitudinal assessment to account for the effects of repeated testing. Another caveat in interpreting the results of operant testing is that cognitive and motor dysfunction can start to appear at comparatively different times and they can influence each other. It is recommended to always include at least one test that relies heavily on motor function, e.g. rotarod test, as well as to assess sensory perception of the stimulus used in the operant equipment (olfactory, vision, auditory, tactile, etc.). The experimental design of operant studies is crucial, as it can significantly impact upon the results obtained. In longitudinal studies, animals are often repeatedly testing in operant tasks and this type of repeated testing has been shown to modify the associated disease phenotype [18]. This leads to the conclusion 
that cognitive training can modify the disease phenotype in mouse models of HD [18, 19] and also has important implications for clinical research. 


\section{Acknowledgements}

E.Y. is supported by a Health and Care Research Wales Health Fellowship award and has also received research funding from the Jacque and Gloria Gossweiler Foundation as well as a previous PhD studentship from the Medical Research Council (MRC), United Kingdom.

A.H. is supported by a scholarship of the Swedish Society for Medical research (SSMF) and a starting grant of the Swedish research council (Vetenskapsradet).

Both authors would like to acknowledge past and present members of the Brain Repair Group at Cardiff University and particularly the contribution of Professor Stephen B. Dunnett who has developed and refined these tasks over many decades. Furthermore, we would like to thank David H. Harrison for providing photographs of the operant equipment and Michael A. Yhnell for proof reading the content of this Chapter. 
Table 1 - Operant tests of cognition in Huntington's disease mice

\begin{tabular}{|c|c|c|}
\hline Operant Test & Cognitive domain & Key Reference (s) \\
\hline Fixed Ratio & $\begin{array}{l}\text { Responding and amenability } \\
\text { in responding }\end{array}$ & {$[19,55,57,80]$} \\
\hline Progressive Ratio & Motivation & {$[19,57,80]$} \\
\hline 5-Choice Serial Reaction Time Task & Attention & {$[18,56,57]$} \\
\hline Serial Implicit Learning Task & $\begin{array}{l}\text { Attention and Implicit } \\
\text { Learning }\end{array}$ & {$[47,57,81,82]$} \\
\hline $\begin{array}{l}\text { Delayed matching and non- } \\
\text { matching to position or sample }\end{array}$ & $\begin{array}{l}\text { Reversal learning and delay } \\
\text { dependent memory }\end{array}$ & {$[18,69,71]$} \\
\hline $\begin{array}{l}\text { The 'Carli' task } \\
\text { (lateralised choice reaction time } \\
\text { task) }\end{array}$ & Response bias & {$[52,74,83]$} \\
\hline Delayed alternation & Working memory & {$[68,84]$} \\
\hline
\end{tabular}




\section{$\underline{\text { References }}$}

1. MacDonald ME, Ambrose CM, Duyao MP, et al., (1993) A novel gene containing a trinucleotide repeat that is expanded and unstable on Huntington's disease chromosomes. Cell. 72:971-983

2. Jacobsen JC, Bawden CS, Rudiger SR, et al., (2010) An ovine transgenic Huntington's disease model. Hum Mol Genet. 19:1873-1882

3. Yang D, Wang C-E, Zhao B, et al., (2010) Expression of Huntington's disease protein results in apoptotic neurons in the brains of cloned transgenic pigs. Hum Mol Genet. 19:3983-3994

$4 . \quad$ Yang S-H, Cheng P-H, Banta $\mathrm{H}$, et al., (2008) Towards a transgenic model of

Huntington's disease in a non-human primate. Nature. 453:921-924

5. von Hörsten S, Schmitt I, Nguyen HP, et al., (2003) Transgenic rat model of

Huntington's disease. Hum Mol Genet. 12:617-624

6. Faber PW, Alter JR, MacDonald ME, et al., (1999) Polyglutamine-mediated dysfunction and apoptotic death of a Caenorhabditis elegans sensory neuron. P Natl Acad Sci. 96:179-184

7. Lunkes A, Mandel J-L, (1998) A cellular model that recapitulates major pathogenic steps of Huntington's disease. Hum Mol Gen. 7:1355-1361

8. Mangiarini L, Sathasivam K, Seller M, et al., (1996) Exon 1 of the HD gene with an expanded CAG repeat is sufficient to cause a progressive neurological phenotype in transgenic mice. Cell. 87:493-506

9. Brooks S, Higgs $G$, Jones L, et al., (2012) Longitudinal analysis of the behavioural phenotype in Hdh (CAG) 150 Huntington's disease knock-in mice. Brain Res Bull. 88:182-188 10. Brooks SP, Janghra N, Workman VL, et al., (2012) Longitudinal analysis of the behavioural phenotype in R6/1 (C57BL/6J) Huntington's disease transgenic mice. Brain Res Bull. 88:94-103

11. Brooks S, Higgs G, Janghra N, et al., (2012) Longitudinal analysis of the behavioural phenotype in YAC128 (C57BL/6J) Huntington's disease transgenic mice. Brain Res Bull. 88:113-120 12. Menalled L, El-Khodor BF, Patry M, et al., (2009) Systematic behavioral evaluation of Huntington's disease transgenic and knock-in mouse models. Neurobiol Dis. 35:319-336

13. Rogers DC, Fisher E, Brown S, et al., (1997) Behavioral and functional analysis of mouse phenotype: SHIRPA, a proposed protocol for comprehensive phenotype assessment. Mamm Genome. 8:711-713

14. Baldo B, Petersén Å: Chapter 35 (2015) Analysis of Nonmotor Features in Murine Models of Huntington Disease A2 - LeDoux, Mark S. Movement Disorders (Second Edition). Boston: Academic Press 583-602.

15. Brooks SP, Dunnett SB, (2009) Tests to assess motor phenotype in mice: a user's guide. Nat Rev Neuro. 10:519-529

16. Crawley JN, (2008) Behavioral phenotyping strategies for mutant mice. Neuron.

$57: 809-818$

17. Yue F, Cheng Y, Breschi A, et al., (2014) A comparative encyclopedia of DNA elements in the mouse genome. Nature. 515:355-364

18. Yhnell E, Lelos MJ, Dunnett SB, et al., (2016) Cognitive training modifies disease symptoms in a mouse model of Huntington's disease. Exp Neuro. 282:19-26

19. Curtin PC, Farrar AM, Oakeshott S, et al., (2015) Cognitive Training at a Young Age Attenuates Deficits in the zQ175 Mouse Model of HD. Front Behav Neuro. 9

20. Huntington G, (1872) Medical and Surgical Reporter. On Chorea:320-321

21. Thompson P, Berardelli A, Rothwell J, et al., (1988) The coexistence of bradykinesia and chorea in Huntington's disease and its implications for theories of basal ganglia control of movement. Brain. 111:223-244

22. Diamond R, White RF, Myers RH, et al., (1992) Evidence of presymptomatic cognitive decline in Huntington's disease. J Clin Exp Neuropsyc. 14:961-975 

deficits in preclinical Huntington's disease. Brain. 121:1329-1341

24. Kirkwood S, Siemers E, Hodes M, et al., (2000) Subtle changes among presymptomatic carriers of the Huntington's disease gene. Journal Neuro, Neurosur PS. 69:773-779

25. Robins Wahlin T-B, Lundin A, Dear K, (2007) Early cognitive deficits in Swedish gene carriers of Huntington's disease. Neuropsychology. 21:31

26. Paulsen J, Langbehn D, Stout J, et al., (2008) Detection of Huntington's disease decades before diagnosis: the Predict-HD study. Journal of Neuro, Neurosur PS. 79:874-880

27. Tabrizi SJ, Langbehn DR, Leavitt BR, et al., (2009) Biological and clinical manifestations of Huntington's disease in the longitudinal TRACK-HD study: cross-sectional analysis of baseline data. The Lancet Neurol. 8:791-801

28. Tabrizi SJ, Scahill RI, Durr A, et al., (2011) Biological and clinical changes in premanifest and early stage Huntington's disease in the TRACK-HD study: the 12-month longitudinal analysis. The Lancet Neuro. 10:31-42

29. Tabrizi SJ, Scahill RI, Owen G, et al., (2013) Predictors of phenotypic progression and disease onset in premanifest and early-stage Huntington's disease in the TRACK-HD study: analysis of 36-month observational data. The Lancet Neuro. 12:637-649

30. Craufurd D, Thompson JC, Snowden JS, (2001) Behavioral changes in Huntington disease. Cogn and Behav Neuro. 14:219-226

31. Thompson JC, Snowden JS, Craufurd D, et al., (2002) Behavior in Huntington's disease: dissociating cognition-based and mood-based changes. Journal Neuropsych Clin N. 14:37-43

32. Lawrence AD, Sahakian B, Rogers R, et al., (1999) Discrimination, reversal, and shift learning in Huntington's disease: mechanisms of impaired response selection. Neuropsychologia. 37:1359-1374

33. Josiassen RC, Curry LM, Mancall EL, (1983) Development of neuropsychological deficits in Huntington's disease. Arch Neurol. 40:791-796

34. Snowden J, Austin N, Sembi S, et al., (2008) Emotion recognition in Huntington's disease and frontotemporal dementia. Neuropsychologia. 46:2638-2649

35. Kipps C, Duggins A, McCusker E, et al., (2007) Disgust and happiness recognition correlate with anteroventral insula and amygdala volume respectively in preclinical Huntington's disease. J Cog Neuro. 19:1206-1217

36. Johnson SA, Stout JC, Solomon AC, et al., (2007) Beyond disgust: impaired recognition of negative emotions prior to diagnosis in Huntington's disease. Brain. 130:1732-1744

37. Helder D, Kaptein A, Van Kempen G, et al., (2001) Impact of Huntington's disease on quality of life. Mov Dis. 16:325-330

38. Ready RE, Mathews M, Leserman A, et al., (2008) Patient and caregiver quality of life in Huntington's disease. Mov Dis. 23:721-726

39. Mitchell AJ, Kemp S, Benito-León J, et al., (2010) The influence of cognitive impairment on health-related quality of life in neurological disease. Acta Neuropsychiatr. 22:2-13

40. Burns A, Folstein S, Brandt J, et al., (1990) Clinical assessment of irritability, aggression, and apathy in Huntington and Alzheimer disease. J Nerv Ment Dis. 178:20-26

41. GUSELLA JF, MACDONALD ME, DUFF K, et al., (2007) Psychiatric Symptoms in Huntington's Disease before Diagnosis: The Predict-HD Study. Commentary. Biol Psych. 62

42. Julien CL, Thompson JC, Wild S, et al., (2007) Psychiatric disorders in preclinical Huntington's disease. J Neuro, Neurosurg Psych. 78:939-943

43. Klöppel S, Stonnington CM, Petrovic $P$, et al., (2010) Irritability in pre-clinical Huntington's disease. Neuropsychologia. 48:549-557

44. Baudic S, Maison P, Dolbeau G, et al., (2006) Cognitive impairment related to apathy in early Huntington's disease. Dement Geriatr Cogn. 21:316-321

45. Paulsen JS, Nehl C, Hoth KF, et al., (2005) Depression and stages of Huntington's disease. Journal Neuropsy Clin Neuro. 17:496-502 
Slaughter JR, Martens MP, Slaughter KA, (2001) Depression and Huntington's disease: prevalence, clinical manifestations, etiology, and treatment. CNS spectrums. 6:306-308,325-326

47. Brooks SP, Jones L, Dunnett SB, (2012) Longitudinal analyses of operant performance on the serial implicit learning task (SILT) in the YAC128 Huntington's disease mouse line. Brain Res Bull. 88:130-136

48. Takao K, Miyakawa T, (2006) Light/dark transition test for mice. JoVE:e104-e104 49. Brasted PJ, Döbrössy MD, Robbins TW, et al., (1998) Striatal lesions produce distinctive impairments in reaction time performance in two different operant chambers. Brain Res Bull. 46:487493

50. Skinner B, (1938) The Behavior of Organisms (Appleton-Century-Crofts, New York). Spaulding, WD, Storms, L., Goodrich, V., and Sullivan, M.(1986), Applications of experimental psychopathology in psychiatric rehabilitation. Schizophrenia Bull. 12:560577

51. Yhnell E, Dunnett SB, Brooks SP, (2016) The utilisation of operant delayed matching and non-matching to position for probing cognitive flexibility and working memory in mouse models of Huntington's disease. J Neuro Meth. 265:72-80

52. Carli M, Robbins T, Evenden J, et al., (1983) Effects of lesions to ascending noradrenergic neurones on performance of a 5-choice serial reaction task in rats; implications for theories of dorsal noradrenergic bundle function based on selective attention and arousal. Behav Brain Res. 9:361-380

53. Robbins T, (2002) The 5-choice serial reaction time task: behavioural pharmacology and functional neurochemistry. Psychopharmacology. 163:362-380

54. Humby T, Laird FM, Davies W, et al., (1999) Visuospatial attentional functioning in mice: interactions between cholinergic manipulations and genotype. Eur J Neuro. 11:2813-2823

55. Bensadoun J-C, Brooks SP, Dunnett SB, (2004) Free operant and discrete trial performance of mice in the nine-hole box apparatus: validation using amphetamine and scopolamine. Psychopharmacology. 174:396-405

56. Trueman RC, Dunnett SB, Jones L, et al., (2012) Five choice serial reaction time performance in the Hdh Q92 mouse model of Huntington's disease. Brain Res Bull. 88:163-170 57. Yhnell E, Dunnett SB, Brooks SP, (2016) A Longitudinal Operant Assessment of Cognitive and Behavioural Changes in the Hdh Q111 Mouse Model of Huntington's Disease. PloS one. 11:e0164072

58. Horner AE, Heath CJ, Hvoslef-Eide M, et al., (2013) The touchscreen operant platform for testing learning and memory in rats and mice. Nature Protocols. 8:1961-1984

59. Morton AJ, Skillings E, Bussey TJ, et al., (2006) Measuring cognitive deficits in disabled mice using an automated interactive touchscreen system. Nature Methods. 3:767-767

60. Clelland C, Choi M, Romberg C, et al., (2009) A functional role for adult hippocampal neurogenesis in spatial pattern separation. Science. 325:210-213

61. McTighe SM, Mar AC, Romberg C, et al., (2009) A new touchscreen test of pattern separation: effect of hippocampal lesions. Neuroreport. 20:881-885

62. Talpos J, McTighe S, Dias R, et al., (2010) Trial-unique, delayed nonmatching-tolocation (TUNL): a novel, highly hippocampus-dependent automated touchscreen test of location memory and pattern separation. Neurobiol Learn Mem. 94:341-352

63. Trueman RC, Brooks SP, Jones L, et al., (2009) Rule learning, visuospatial function and motor performance in the Hdh Q92 knock-in mouse model of Huntington's disease. Behav Brain Res. 203:215-222

64. Dunnett SB, Fuller A, Rosser AE, et al., (2012) A novel extended sequence learning task (ESLeT) for rodents: validation and the effects of amphetamine, scopolamine and striatal lesions. Brain Res Bull. 88:237-250

65. Kubota K, Niki H, (1971) Prefrontal cortical unit activity and delayed alternation performance in monkeys. J Neurophys

66. Jacobsen CF, Nissen H, (1937) Studies of cerebral function in primates. IV. The effects of frontal lobe lesions on the delayed alternation habit in monkeys. J Comp Psych. 23:101 
67.

Dunnett SB, Nathwani F, Brasted PJ, (1999) Medial prefrontal and neostriatal lesions disrupt performance in an operant delayed alternation task in rats. Behav Brain Res. 106:13-28

68. Trueman RC, Jones L, Dunnett SB, et al., (2012) Early onset deficits on the delayed alternation task in the Hdh Q92 knock-in mouse model of Huntington's disease. Brain Res Bull. 88:156-162

69. Dunnett SB, Rogers DC, Jones GH, (1989) Effects of nucleus basalis magnocellularis lesions in rats on delayed matching and non-matching to position tasks. E J Neuro. 1:395-406

70. Dunnett SB, (1985) Comparative effects of cholinergic drugs and lesions of nucleus basalis or fimbria-fornix on delayed matching in rats. Psychopharmacology. 87:357-363

71. D'amato M, (1973) Delayed matching and short-term memory in monkeys. Psych Learn Motiv. 7:227-269

72. Carli M, Evenden J, Robbins T, (1985) Depletion of unilateral striatal dopamine impairs initiation of contralateral actions and not sensory attention. Nature 313.6004: 679-682.

73. Döbrössy MD, Dunnett SB, (1997) Unilateral striatal lesions impair response execution on a lateralised choice reaction time task. Behav Brain Res. 87:159-171

$74 . \quad$ Döbrössy MD, Dunnett SB, (1998) Striatal grafts alleviate deficits in response execution in a lateralised reaction time task. Brain Res Bull. 47:585-593

75. Dowd E, Dunnett SB, (2005) Comparison of 6-hydroxydopamine-induced medial forebrain bundle and nigrostriatal terminal lesions in a lateralised nose-poking task in rats. Behav Brain Res. 159:153-161

76. Mayer E, Brown V, Dunnett S, et al., (1992) Striatal graft-associated recovery of a lesion-induced performance deficit in the rat requires learning to use the transplant. E J Neuro. 4:119-126

77. Van Raamsdonk JM, Metzler M, Slow E, et al., (2007) Phenotypic abnormalities in the YAC128 mouse model of Huntington disease are penetrant on multiple genetic backgrounds and modulated by strain. Neurobio Dis. 26:189-200

78. Tucci V, Hardy A, Nolan PM, (2006) A comparison of physiological and behavioural parameters in C57BL/6J mice undergoing food or water restriction regimes. Behav Brain Res. 173:2229

79. Duan W, Guo Z, Jiang H, et al., (2003) Dietary restriction normalizes glucose metabolism and BDNF levels, slows disease progression, and increases survival in huntingtin mutant mice. Procs Natl Acad Sci. 100:2911-2916

80. Oakeshott S, Port R, Cummins-Sutphen J, et al., (2012) A mixed fixed ratio/progressive ratio procedure reveals an apathy phenotype in the BAC HD and the $z_{-} \mathrm{Q} 175 \mathrm{KI}$ mouse models of Huntington's disease. PLOS Currents Huntington Disease

81. Trueman RC, Brooks SP, Jones L, et al., (2007) The operant serial implicit learning task reveals early onset motor learning deficits in the HdhQ92 knock-in mouse model of Huntington's disease. E J Neuro. 25:551-558

82. Trueman RC, Brooks SP, Jones L, et al., (2008) Time course of choice reaction time deficits in the Hdh Q92 knock-in mouse model of Huntington's disease in the operant Serial Implicit Learning Task (SILT). Behav Brain Res. 189:317-324

83. Dowd E, Dunnett SB, (2004) Deficits in a lateralized associative learning task in dopamine-depleted rats with functional recovery by dopamine-rich transplants. E J of Neuro. 20:1953-1959

84. Dunnett SB, White A, (2006) Striatal grafts alleviate bilateral striatal lesion deficits in operant delayed alternation in the rat. Exper Neuro. 199:479-489 